\title{
Pyloric Stenos is of Infancy-The Great Mystery Unravels
}

\author{
I.M.Rogers FRCS, FRCP, PGCMed.Edu*
}

*Corresponding Author: I.M.Rogers, 46 Whitburn Road, Cleadon, Sunderland SR67QS, United Kingdom, E-mail: irogers2000@hotmail.com

\section{BOOK REVIEW}

Infantile pyloric stenosis is a very interesting disease. Although nothing has changed regarding the concept of surgical treatment since the introduction of the technique of pyloromyotomy by Fredet and Ramstedt, the aetiopathogenesis is still enigmatic.

Many features of the disease are confusing and still needs logic explanation, including the delayed presentation, the male preponderance, and the unexplained heredity pattern.

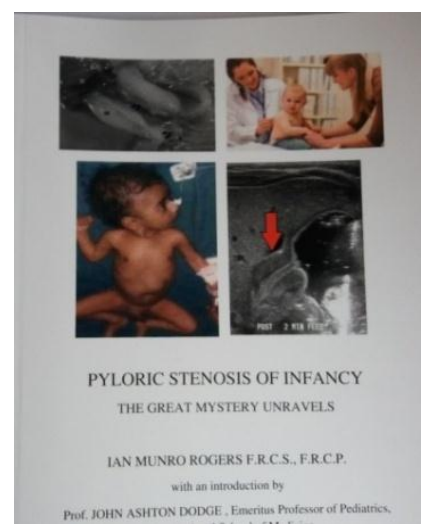

The Book "PYLORIC STENOSIS OF INFANCY-THE GREAT MYSTERY UNRAVELS." by Ian Rogers answers all these questions and more. The book is an in-depth coverage of the problem and is the result of years of deep thinking and investigations.
Dr. Rogers presents a very nice sequence in the book starting with an interesting review of the historical landmarks of the theories of causation; the $1^{\text {st }}$ attempts of medical treatment and the evolution of the time-honored approach of pyloromyotomy by Fredet and Ramstedt.

The cornerstone of Dr. Rogers theory is the hyperacidity and work overload on the pyloric sphincter. If you test this theory with various aspects of the disease you will find it is very fitting. This has many clinical implications including the treatment of perioperative alkalosis and the reversibility of some of the early cases with acid-reducing medications. The book displays the interesting analogy between pyloric stenos is of infancy and duodenal ulcer disease of adulthood.

In the absence of a credible alternative explanation, it is the opinion of this reviewer that the Primary Hyperacidity theory as here detailed and debated is likely to be the true cause.

The book should interest a large group of readership including pediatric surgeons, pediatricians, geneticists, and medical students. The Book is available for purchase at Amazon Books U.k and that all profits from the Sale go to a charity- the Safe Water Trust.

Citation: I.M.Rogers. Pyloric Stenos is of Infancy-The Great Mystery Unravels. ARC Journal of Pediatrics.2019; 5(1):1. doi:dx.doi.org/10.20431/2455-5711.0501001.

Copyright: (c) 2019 Authors. This is an open-access article distributed under the terms of the Creative Commons Attribution License, which permits unrestricted use, distribution, and reproduction in any medium, provided the original author and source are credited. 